\title{
Conocimiento y uso del lenguaje matemático en la formación inicial de docentes en matemáticas
}

\section{Knowledge and use of mathematical language in initial teacher training in mathematics}

\author{
César Augusto Hernández-Suárez ${ }^{1}$ \\ Raúl Prada-Núñez ${ }^{2}$ \\ Audin Aloiso Gamboa-Suárez ${ }^{3}$
}

Recibido: septiembre 29 de 2016

Aceptado: diciembre 28 de 2016

\section{Resumen}

Este artículo analiza los niveles de competencias en el conocimiento y uso del lenguaje matemático que poseen y emplean los estudiantes durante su formación inicial en la carrera de docencia en matemáticas, en una universidad pública colombiana. La investigación se propone establecer el nivel de conocimiento y uso del lenguaje matemático entre estos estudiantes, qué nivel de habilidad matemática emplean y su competencia para comunicar por escrito sus conocimientos. El diseño de la investigación es descriptivo y de campo, para lo cual se empleó como muestra un grupo de 92 estudiantes de un programa de formación inicial de docentes que cursan las asignaturas del eje disciplinar de matemáticas. Sobre la base de la resolución escrita de problemas se determinó el nivel de conocimiento y uso del lenguaje matemático empleado por los estudiantes. Los resultados muestran desconocimiento y mal uso del lenguaje y la simbología matemática, de las reglas de la lógica y escasa capacidad de razonamiento, lo cual genera dificultades para comprender y resolver problemas matemáticos. Se determinó asimismo que los estudiantes presentan mayores dificultades en la traducción del lenguaje cotidiano al formal, en la identificación de objetos matemáticos y en el trabajo con conceptos que ameritan demos- traciones lógicas. Los estudiantes poseen un nivel insuficiente de conocimiento y uso del lenguaje matemático, habilidad matemática también baja y mayor competencia para descifrar el lenguaje matemático hacia el verbal que en cifrar lenguaje verbal en lenguaje matemático.

Palabras Clave: enseñanza de las matemáticas, lenguaje matemático, formación inicial, competencias del docente.

\begin{abstract}
This article analyses the levels of knowledge and use of mathematical language that students possess and employ during their initial teaching training for the program of mathematics in a Colombian public university. The research specifically proposes to establish the level of knowledge and use of mathematical language among these students, what level of math skills they use and their competence to communicate in writing their knowledge. The research design is descriptive and in field, where a sample group of 92 students from an initial teacher training program who were enrolled in subjects of discipline axis of Maths were taken into consideration. On the basis of the written problem solving level, the knowledge and use of mathematical language used by students was
\end{abstract}

\footnotetext{
1 Licenciado en Matemáticas y Computación, Doctor en Educación, Universidad Francisco de Paula Santander, Colombia. E-mail: cesaraugusto@ufps.edu.co

2 Licenciado en Matemáticas y Computación, Magíster en Matemática, Universidad Francisco de Paula Santander, Colombia. E-mail: raulprada@ufps.edu.co

3 Licenciado en Música, Doctor en Ciencias de la Educación, Universidad Francisco de Paula Santander, Colombia. E-mail: audingamboa@ufps.edu.co
} 
determined. The results show ignorance and misuse of language and mathematical symbols, rules of logic and reasoning are limited, which creates difficulties in understanding and solving mathematical problems. It was also determined that students have greater difficulties in translating the everyday language to formal, in identifying mathematical objects and work with concepts that merit logical demonstrations. Students also have an insufficient level of knowledge and use of mathematical language, a low mathematical ability and more competition to decipher the mathematical language to verbal than verbal language to encrypt mathematical language.

Keywords: teaching mathematics, mathematical language, initial training, teacher skills.

\section{Introducción}

La formación de docentes de matemática competentes se inicia, necesariamente, en el dominio y comprensión por parte de los futuros docentes de los contenidos que constituyen la materia de su enseñanza, la destreza para su apropiado manejo y aplicación y la habilidad para comunicar convenientemente el contenido matemático. La realidad de la formación inicial de docentes es muy variada. En Latinoamérica, tal y como lo afirma Gómez-Chacón:

un porcentaje de estudiantes que se preparan para ser docentes de matemáticas que no poseen las destrezas y competencias matemáticas suficientes, que se deberían desarrollar a lo largo de la escolaridad (comenzando en primaria y profundizando en secundaria) y son esenciales para los estudios superiores: comprensión conceptual de las nociones matemáticas elementales, destrezas procedimentales en los procesos de construcción matemático, pensamiento estratégico (formular, representar y resolver problemas), capacidades para comunicar y explicar matemáticamente y actitudes positivas ante la propia capacidad matemática. $(2005$, p. 28)
Por todo ello, en la formación inicial de los docentes en matemáticas resultan esenciales tanto la inducción hacia el conocimiento de los contenidos como el desarrollo de competencias para su manejo, aplicación y comunicación. Sin embargo, la competencia comunicativa no se refiere únicamente al despliegue eficaz de estrategias didácticas durante el ejercicio profesional, sino también (y con anterioridad) a la capacidad para demostrar dominio adecuado de las nociones propias de la ciencia y sus disciplinas y establecer las relaciones que existen entre ellas con suficiente solvencia. Pero, como lo afirma Vilaró, "es muy común que la Educación Matemática se desarrolle en los centros de formación inicial, no integrada a la formación científica" $(2005$, p. 60).

En este sentido, la evaluación de los contenidos del eje curricular durante la formación inicial no debe limitarse a la medición de la apropiación por parte del estudiante del conocimiento específico de cada una de las asignaturas que lo constituyen; las estrategias de evaluación, en efecto, resultan una valiosa oportunidad para la percepción de la capacidad que poseen y van desarrollando los futuros docentes para comunicar los saberes que adquieren. En efecto, la comunicación de los contenidos de matemática se contempla en la actualidad como pieza fundamental del aprendizaje y está considerada como una competencia profesional indiscutida dentro de la formación inicial docente (Vargas., \& Giménez, 2012). Dado que la comunicación en el desarrollo de los contenidos matemáticos es un valioso determinante de éxito de la propia actuación docente en el aula, resulta incuestionable la necesidad de fortalecer este aspecto durante la formación inicial.

El lenguaje, según Godino (citado por Sepúlveda-Delgado 2015), se encuentra "constituido por los términos, las expresiones, las notaciones, las gráficas. En un texto vienen dados en forma escrita o gráfica, pero en el trabajo matemático pueden usarse otros registros (oral, gestual). Mediante el lenguaje (ordinario y matemático) se describen otros objetos lingüísticos". 
Por otra parte la autora afirma que:

Los diversos medios de expresión, lenguajes, desempeñan el doble papel de instrumentos de trabajo matemáticos y de representación de los restantes objetos matemáticos; permite describir los objetos y procesos matemáticos que intervienen en la realización de las prácticas, así como los que emergen de ellas (situaciones, lenguajes, conceptos, propiedades, procedimientos y argumentos) (Sepúlveda-Delgado, 2015, p. 39).

Queda claro que la comprensión del lenguaje matemático, al igual que el resto de los procesos matemáticos fundamentales en la formación del estudiante, tales como: la resolución de problemas, el razonamiento y la demostración, las conexiones y representaciones, se deben incluir en el currículo de matemáticas por medio de un desarrollo continuo del lenguaje y la simbología, lo cual permitirá posteriormente la adecuada comunicación de las ideas matemáticas. Es por ello que los programas de formación inicial del docente deben incluir estos procesos, tanto desde la perspectiva del dominio de los contenidos como con la capacidad de comunicarlos.

La importancia del desarrollo de la capacidad de comunicarse matemáticamente, del papel del lenguaje en el aprendizaje de las matemáticas y la didáctica de la disciplina ha sido asimismo objeto de investigaciones en el último tiempo. Ejemplo de ello son los trabajos de Godino, Batanero y Font (2004); de Cerón, Mesa y Rojas (2012); de Aldana-Bermúdez y López-Mesa (2016), o de Niss $(1999,2003)$.

En cuanto a los lineamientos curriculares de matemáticas, es sabido que entre los procesos a desarrollar y que forman parte del currículo planteados por el Ministerio de Educación Nacional aparece la comunicación matemática. De acuerdo con la concepción del propio Ministerio, la comunicación matemática implica "reconocer el lenguaje propio de las matemáticas, usar las nociones y procesos matemáticos en la comunicación, reconocer sus significados, expresar, interpretar y evaluar ideas matemáticas, construir, interpretar y ligar representaciones, producir y presentar" (Ministerio de Educación Nacional, 2006a). Todos estos procesos cognitivos y reflexivos son básicos para guiar la acción docente en el aula. Pero los lineamientos del Ministerio amplían y profundizan el desarrollo de competencias en área dejando claro que se deben:

Utilizar diferentes registros de representación o sistemas de notación simbólica para crear, expresar y representar ideas matemáticas; para utilizar y transformar dichas representaciones y, con ellas, formular y sustentar puntos de vista. Es decir, dominar con fluidez distintos recursos y registros del lenguaje cotidiano y de los distintos lenguajes matemáticos. (Ministerio de Educación Nacional, 2006b, p. 51)

En este contexto, autores como (Niss, 1999) sitúan una serie de competencias ligadas con la habilidad para utilizar el lenguaje matemático, entre las que se encuentran representar objetos y situaciones matemáticas; utilizar símbolos y formalismos matemáticos y comunicar en, con y acerca de las matemáticas (González-Marí, 2004).

Según los Principios y Estándares para la Matemática Escolar (National Council of Teachers of Mathematics, NCTM, 2003) la comunicación es una parte esencial de las matemáticas y la educación. El estándar de comunicación es primordial para todos los programas de enseñanza y en todas las etapas se debería formar a los estudiantes para organizar y consolidar el pensamiento matemático a través de la comunicación; expresar el pensamiento matemático con coherencia y claridad a los compañeros, profesores y otras personas; analizar y evaluar las estrategias y el pensamiento matemático de los demás y usar el lenguaje de las matemáticas para expresar ideas matemáticas con precisión (National Council of Teachers of Mathematics, 2003). 
Investigaciones como la de Piratoba y Rojas (2014) estudian cómo cambian las concepciones que tienen los estudiantes en un programa de formación inicial para profesores de matemáticas sobre la naturaleza de las matemáticas, su forma de aprender y enseñar. Las autoras describen la capacidad que tienen los estudiantes de formación inicial en la carrera docente de matemáticas para comunicar, a través de la resolución de problemas, su dominio del lenguaje matemático a medida que estos avanzan en las asignaturas del eje disciplinar de matemáticas. Esta capacidad deviene, así, en un modo de dimensionar la evaluación de contenidos y establecer los niveles de competencia en el conocimiento y uso del lenguaje matemático.

\section{Marco metodológico}

\subsection{Método}

La naturaleza de la investigación es cuantitativa con carácter descriptivo, pues a partir de métodos estadísticos de resumen y tablas de frecuencia, aplicados sobre un conjunto de datos obtenidos de un cuestionario, se busca caracterizar los niveles de competencia en el conocimiento y uso del lenguaje matemático de docentes en formación inicial en matemática. Se trata de un estudio de campo por cuanto los datos se recogen in situ, en este caso en la Facultad de Educación, Artes y Humanidades de la Universidad Francisco de Paula Santander, UFPS, en la ciudad de Cúcuta, Colombia.

El diseño investigativo consiste en un estudio transversal, pues los datos se colectaron en un solo momento, y se centró en un proceso descriptivo con la finalidad de analizar el nivel de las competencias del lenguaje matemático empleado en las producciones escritas de los estudiantes.

\subsection{Muestra}

Se trabajó con todos los estudiantes que en ese momento cursaban las asignaturas geometría superior, lógica, teoría de conjuntos, teoría de números, álgebra abstracta, análisis matemático y topología. La muestra estuvo constituida por los 92 estudiantes matriculados durante el segundo semestre de 2014 en el programa Licenciatura en Matemáticas de la UFPS, cuya duración es de 10 semestres académicos. En efecto, la población referenció el marco muestral y de manera intencional se consideraron para el estudio la totalidad de los estudiantes; en consecuencia, se puede hablar de un censo poblacional (Méndez, 2007). La elección, de acuerdo con los criterios del investigador, fue de tipo casual o incidental, por lo tanto, no aleatoria. Los estudiantes, en efecto, fueron elegidos por cuanto para el momento de la recolección de datos estaban cursando las asignaturas del eje de formación en matemáticas.

\subsection{Instrumento}

Con el fin de obtener una información organizada y sistemática sobre el lenguaje y la comunicación matemática, se adaptó un cuestionario (Hernández, 2009, 2012), que permitió determinar los niveles de competencia en el conocimiento y uso del lenguaje matemático. Este instrumento consta de 6 ítems, a través de los cuales se indagó sobre cuestiones concretas relativas al lenguaje matemático y la comunicación en matemáticas. El instrumento fue evaluado por tres expertos en el área y se adoptaron las sugerencias que hicieron para su mejoramiento y confección final. Posteriormente se realizó una prueba piloto con un grupo de estudiantes ajenos a la muestra definitiva; esta prueba reportó una confiabilidad alta. Los descriptores de competencia del instrumento se muestran en la Tabla 1. 


\begin{tabular}{|c|c|c|c|c|}
\hline \multirow{2}{*}{ Ítem } & \multirow[b]{2}{*}{ Variable } & \multicolumn{2}{|c|}{ Descriptores de competencia analizados } & \multirow{2}{*}{ Actividad a desarrollar } \\
\hline & & MEN - ICFES & PISA (González Mari) & \\
\hline 1 & & $\begin{array}{l}\text { Utilizar sistemas de nota- } \\
\text { ción simbólica. }\end{array}$ & $\begin{array}{l}\text { Utilizar y manipular sen- } \\
\text { tencias y expresiones } \\
\text { que contienen símbolos } \\
\text { y fórmulas. }\end{array}$ & $\begin{array}{l}\text { Los estudiantes deben } \\
\text { identificar el nombre, uso y } \\
\text { significado de una serie de } \\
\text { símbolos matemáticos. }\end{array}$ \\
\hline 2 & USO & $\begin{array}{l}\text { Usar las nociones y proce- } \\
\text { sos matemáticos en la co- } \\
\text { municación y reconocer } \\
\text { sus significados. }\end{array}$ & $\begin{array}{l}\text { Decodificar e interpre- } \\
\text { tar lenguaje matemá- } \\
\text { tico simbólico y formal } \\
\text { y comprender sus rela- } \\
\text { ciones con el lenguaje } \\
\text { natural. }\end{array}$ & $\begin{array}{l}\text { Los estudiantes deben co- } \\
\text { nocer el significado de una } \\
\text { serie de palabras relaciona- } \\
\text { das con nociones y proce- } \\
\text { sos matemáticos. }\end{array}$ \\
\hline 3,4 & & $\begin{array}{l}\text { Dominar con fluidez dis- } \\
\text { tintos recursos y registros } \\
\text { del lenguaje cotidiano y } \\
\text { de los distintos lenguajes } \\
\text { matemáticos. }\end{array}$ & $\begin{array}{l}\text { Traducir del lenguaje } \\
\text { natural al lenguaje sim- } \\
\text { bólico/formal y vicever- } \\
\text { sa. }\end{array}$ & $\begin{array}{l}\text { Se proporcionan expresio- } \\
\text { nes escritas en lenguaje } \\
\text { formal matemático para } \\
\text { que los estudiantes las tra- } \\
\text { duzcan al lenguaje natural y } \\
\text { viceversa. }\end{array}$ \\
\hline 5 & CONOCIMIENTO & $\begin{array}{l}\text { Construir, interpretar y li- } \\
\text { gar representaciones. }\end{array}$ & $\begin{array}{l}\text { Comprender, utilizar, } \\
\text { decodificar e interpre- } \\
\text { tar diferentes clases de } \\
\text { representaciones de } \\
\text { objetos, fenómenos y si- } \\
\text { tuaciones matemáticas } \\
\text { y distinguir entre ellas. }\end{array}$ & $\begin{array}{l}\text { Se proporciona a los es- } \\
\text { tudiantes varios ejemplos } \\
\text { de simbología, notación, } \\
\text { expresiones y objetos ma- } \\
\text { temáticos que deben iden- } \\
\text { tificar y diferenciar según } \\
\text { sean enunciados, símbolos } \\
\text { u objetos matemáticos. }\end{array}$ \\
\hline 6 & & $\begin{array}{l}\text { Expresar, interpretar y } \\
\text { evaluar ideas matemáti- } \\
\text { cas. }\end{array}$ & $\begin{array}{l}\text { Comprender la natura- } \\
\text { leza y las reglas de los } \\
\text { sistemas matemáticos } \\
\text { formales (desde ambos } \\
\text { puntos de vista: sintác- } \\
\text { tico y semántico). }\end{array}$ & $\begin{array}{l}\text { Se proporciona a los estu- } \\
\text { diantes una serie de defi- } \\
\text { niciones matemáticas para } \\
\text { que identifiquen si están } \\
\text { correctamente formuladas } \\
\text { y, en caso contrario, pro- } \\
\text { poner una definición apro- } \\
\text { piada corrigiendo los datos } \\
\text { erróneos de la definición } \\
\text { preliminar. }\end{array}$ \\
\hline
\end{tabular}

Tabla 1. Descriptores de competencia del instrumento.

Este instrumento se aplicó individualmente a cada uno de los estudiantes y para su resolución contaron con un máximo de dos horas. Se realizó un análisis estadístico de la información recolectada utilizando el paquete estadístico SPSS 23. Se calculó la proporción de aciertos de la prueba mediante la asignación de una valoración a cada opción de respuesta de naturaleza categórica a cada ítem (1 incorrecto, 2 regular y 3 correcto) Con este fin se generó una escala de niveles con las características contenidas en la Tabla 2: 


\begin{tabular}{cl}
\hline Escala & Desempeño \\
\hline Insuficiente & $\begin{array}{c}\text { Desconoce símbolos, objetos y lenguaje matemáticos. Presenta dificultad para pasar del len- } \\
\text { guaje natural al lenguaje matemático y viceversa. }\end{array}$ \\
$\begin{array}{cl}\text { Aceptable } & \text { Reconoce parcialmente una gama limitada de símbolos, objetos y lenguaje matemáticos. } \\
& \begin{array}{l}\text { Presenta algunas dificultades para pasar del lenguaje natural al lenguaje matemático y vi- } \\
\text { ceversa. }\end{array} \\
\text { Sobresaliente } & \text { Reconoce y utiliza una amplia gama de símbolos, objetos y lenguaje matemáticos. No pre- } \\
80-100 \% \text { de aciertos } & \text { senta dificultad para pasar del lenguaje natural al lenguaje matemático y viceversa. }\end{array}$ \\
\hline
\end{tabular}

Tabla 2. Niveles de conocimiento y uso del lenguaje matemático y de competencia en la comunicación matemática.

\section{Resultados y discusión}

\subsection{Identificación de símbolos}

Con respecto a la variable Uso del Lenguaje Matemático, la Tabla 3 muestra las respuestas relativas a la identificación de símbolos en relación con conceptos básicos de los temas de las distintas asignaturas, relacionados con Teoría de conjuntos y de Lógica proposicional. Se observa una li- gera diferencia, aproximadamente del $4 \%$, entre el porcentaje de respuestas correctas a favor de la identificación de los símbolos relacionados con la Teoría de Conjuntos, con respecto a los símbolos de Lógica proposicional: $46,7 \%$ vs $42,4 \%$. En las respuestas relacionadas con la lógica proposicional existe un porcentaje de desaciertos del 15,2\%. De forma global se aprecia que el número de respuestas correctas fue alto, pues alcanza aproximadamente el $90 \%$.

\begin{tabular}{|c|c|c|c|c|c|}
\hline \multicolumn{3}{|c|}{$\begin{array}{l}\text { Correcto } \\
\text { Incorrecto }\end{array}$} & \multicolumn{2}{|c|}{ Respuesta } & \multirow{2}{*}{$\begin{array}{c}\text { Total } \\
100.0 \%\end{array}$} \\
\hline \multirow{5}{*}{$\begin{array}{l}\text { Identificación de } \\
\text { Símbolos }\end{array}$} & \multirow{2}{*}{ Conjuntos } & $\begin{array}{l}\text { \% dentro de la Teoría de } \\
\text { Conjuntos }\end{array}$ & $93.5 \%$ & $6.5 \%$ & \\
\hline & & $\begin{array}{l}\% \text { del total de respuestas de } \\
\text { Ident. de Símbolos }\end{array}$ & $46.7 \%$ & $3.3 \%$ & $50.0 \%$ \\
\hline & \multirow{3}{*}{$\begin{array}{l}\text { Lógica } \\
\text { Proposicional }\end{array}$} & $\begin{array}{l}\text { \% dentro de las respuestas } \\
\text { de Lógica Proposicional }\end{array}$ & $84.8 \%$ & $15.2 \%$ & $100.0 \%$ \\
\hline & & $\begin{array}{l}\text { \% del total de respuestas de } \\
\text { Ident. de Símbolos }\end{array}$ & $42.4 \%$ & $7.6 \%$ & $50.0 \%$ \\
\hline & & $\%$ total & $89.1 \%$ & $10.9 \%$ & $100.0 \%$ \\
\hline
\end{tabular}

Tabla 3. Uso del Lenguaje Matemático: Identificación de símbolos.

Al evaluar la posible relación de dependencia entre las variables se encuentra un valor de Chi-cuadrado de 3,59 con un grado de libertad y un $p$-valor asociado $p=0,06>0,05$, lo cual permite concluir que la respuesta no presenta ninguna relación o asociación con los contenidos referidos a los temas de Teoría de Conjuntos o la Lógica proposicional y que, por lo tanto, son independientes.

En relación a la variable Conocimiento del Lenguaje Matemático, en la Tabla 4 se muestran las respuestas correspondientes a las evaluaciones en la definición de términos con argumentos lógicos, 
asociadas a demostraciones lógicas. La intención en este ítem era que el estudiante definiera cada una de estas palabras y, de acuerdo con la definición proporcionada, se pudiera evaluar tanto el nivel de conocimientos como sus hábitos demostrativos. El porcentaje de respuestas correctas es superior en casi 10 puntos en las definiciones relacionadas con argumentos lógicos en comparación con las demostraciones lógicas (41,3\% vs $32,9 \%)$.
En cuanto a las respuestas incorrectas el porcentaje más alto se registró en los argumentos lógi$\cos (35,9 \%)$, que resultó similar al de las respuestas regulares de las demostraciones lógicas $(35,4 \%)$. En términos globales la distribución de respuestas correctas e incorrectas en lo que a definición de términos respecta fue similar, con una leve superioridad de las respuestas correctas: $37,4 \%$ vs $33,9 \%$.

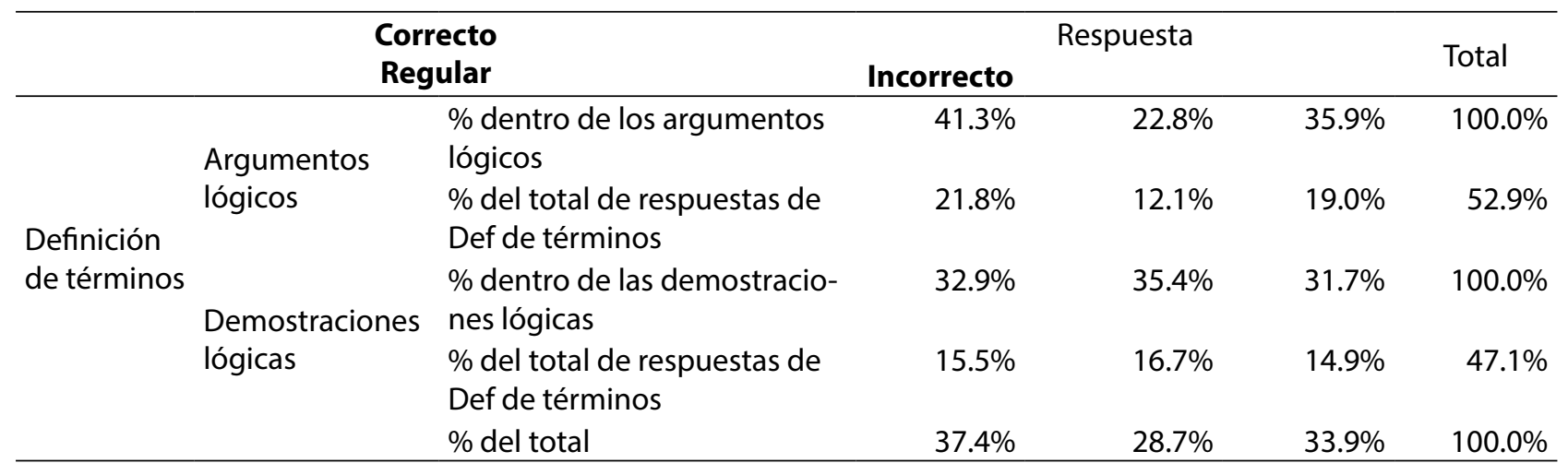

Tabla 4. Conocimiento del Lenguaje Matemático: Definición de términos.

Un valor de Chi-cuadrado de 3,40 con dos grados de libertad y un $p$-valor asociado $p=0,18>0,05$, permiten afirmar que la respuesta no presenta ninguna relación con los tipos de definiciones asociados a argumentos o demostraciones lógicas y que, por lo tanto, son independientes.

Como parte de los aspectos relacionados con el Conocimiento del Lenguaje Matemático, la Tabla 5 muestra la traducción de expresiones del lenguaje formal al cotidiano y viceversa. Se evidenció un número importante de 'no respuestas', que alcanza aproximadamente el $9 \%$ en términos globales. Esta distribución resultó más alta, 12\%, cuando se trataba de una traducción del lenguaje cotidiano al formal. Las respuestas correctas predominaron cuando el ejercicio de traducción se hizo del lenguaje formal al cotidiano, $56,5 \%$, sobre los puntajes de traducción del lenguaje cotidiano al formal, 29,3\%. Ello trajo consigo un mayor número de respuestas claramente incorrectas en la traducción de lo cotidiano a lo formal, 10,9\%, y en consecuencia casi la mitad de respuestas regulares en esta categoría, con 47,8\%. En forma global se observa una cercanía entre los porcentajes de respuestas regulares $(40,8 \%)$ y correctas $(42,9 \%)$. Además, la tabla 6 también muestra la heterogeneidad en la distribución de las respuestas obtenidas, reflejando de manera clara mayor porcentaje de respuestas incorrectas en la traducción del lenguaje cotidiano al formal, y solo mayor número de respuestas regulares en el caso contrario. Un valor de Chi-cuadrado de 14,98 con tres grados de libertad y un $p$-valor asociado $p=0,00<0,05$, permite afirmar que la respuesta está relacionada con el tipo de traducción o cambio de registro semiótico, bien sea del lenguaje cotidiano al formal o del formal al cotidiano. 


\begin{tabular}{|c|c|c|c|c|c|c|c|}
\hline & \multirow{2}{*}{\multicolumn{2}{|c|}{$\begin{array}{l}\text { No Responde } \\
\text { Incorrecto }\end{array}$}} & \multicolumn{4}{|c|}{ Respuesta } & \multirow{3}{*}{$\begin{array}{c}\text { Total } \\
100.0 \%\end{array}$} \\
\hline & & & Regular & Correcto & & & \\
\hline \multirow{5}{*}{ Traducción } & Del lenguaje & $\begin{array}{l}\% \text { de lenguaje for- } \\
\text { mal al cotidiano }\end{array}$ & $5.4 \%$ & $4.3 \%$ & $33.7 \%$ & $56.5 \%$ & \\
\hline & cotidiano & $\begin{array}{l}\% \text { del total de } \\
\text { traducción }\end{array}$ & $2.7 \%$ & $2.2 \%$ & $16.8 \%$ & $28.3 \%$ & $50.0 \%$ \\
\hline & \multirow{3}{*}{$\begin{array}{l}\text { Del lenguaje } \\
\text { cotidiano al } \\
\text { formal }\end{array}$} & $\begin{array}{l}\% \text { de lenguaje coti- } \\
\text { diano al formal }\end{array}$ & $12.0 \%$ & $10.9 \%$ & $47.8 \%$ & $29.3 \%$ & $100.0 \%$ \\
\hline & & $\begin{array}{l}\% \text { del total de } \\
\text { traducción }\end{array}$ & $6.0 \%$ & $5.4 \%$ & $23.9 \%$ & $14.7 \%$ & $50.0 \%$ \\
\hline & & $\%$ del total & $8.7 \%$ & $7.6 \%$ & $40.8 \%$ & $42.9 \%$ & $100.0 \%$ \\
\hline
\end{tabular}

Tabla 5. Conocimiento del Lenguaje Matemático: Cruce entre traducción y respuesta.

\subsection{Identificación de enunciados y objetos}

En relación con el Uso del lenguaje, la Tabla 6 muestra las respuestas obtenidas al identificar y diferenciar los ítems que hacían referencia a enunciados matemáticos, símbolos matemáticos u objetos matemáticos. Las respuestas correctas se registraron principalmente en la identificación de enunciados matemáticos con $62 \%$, y de símbolos matemáticos con $56,5 \%$. En la identificación de objetos matemáticos las respuestas incorrectas fueron altas y alcanzaron el $67,4 \%$. De forma glo- bal se puede hablar de un porcentaje equivalente de respuestas correctas en aproximadamente la mitad del grupo. Igualmente, en la tabla 7 se aprecia esta relación, destacándose el mayor número de respuestas correctas en la identificación de enunciados y símbolos matemáticos. Un valor de Chi-cuadrado de 17,94 con dos grados de libertad y un $p$-valor asociado $p=0,00<0,05$, permite aceptar qué respuesta está relacionada con la identificación de un tipo de elemento en particular, bien sea que se trate de enunciados, símbolos u objetos.

\begin{tabular}{|c|c|c|c|c|c|}
\hline \multicolumn{3}{|c|}{$\begin{array}{l}\text { Incorrecto } \\
\text { Correcto }\end{array}$} & \multicolumn{2}{|c|}{ Respuesta } & \multirow{2}{*}{$\begin{array}{l}\text { Total } \\
100.0 \%\end{array}$} \\
\hline \multirow{7}{*}{$\begin{array}{l}\text { Identificación } \\
\text { de Enunciados y } \\
\text { Objetos }\end{array}$} & \multirow{4}{*}{ Objeto Matemático } & \% de Enunciado Matemático & $38.0 \%$ & $62.0 \%$ & \\
\hline & & $\begin{array}{l}\% \text { del total de Identificación de } \\
\text { objetos }\end{array}$ & $12.7 \%$ & $20.7 \%$ & $33.3 \%$ \\
\hline & & $\begin{array}{l}\text { \% de Objeto } \\
\text { Matemático }\end{array}$ & $67.4 \%$ & $32.6 \%$ & $100.0 \%$ \\
\hline & & $\begin{array}{l}\% \text { del total de Identificación de } \\
\text { objetos }\end{array}$ & $22.5 \%$ & $10.9 \%$ & $33.3 \%$ \\
\hline & \multirow{3}{*}{$\begin{array}{l}\text { Símbolo } \\
\text { Matemático }\end{array}$} & $\begin{array}{l}\text { \% de Símbolo } \\
\text { Matemático }\end{array}$ & $43.5 \%$ & $56.5 \%$ & $100.0 \%$ \\
\hline & & $\begin{array}{l}\% \text { del total de Identificación de } \\
\text { objetos }\end{array}$ & $14.5 \%$ & $18.8 \%$ & $33.3 \%$ \\
\hline & & $\%$ del total & $49.6 \%$ & $50.4 \%$ & $100.0 \%$ \\
\hline
\end{tabular}

Tabla 6. Respuestas asociadas a la identificación de enunciados, objetos y símbolos matemáticos. 


\subsection{Nivel de lenguaje matemático}

Al asignar peso a cada opción de respuesta y a cada ítem se puede calcular un promedio ponderado de la prueba, considerando el porcentaje de respuestas correctas y el número de aciertos reflejados en un valor relativo global. Tal promedio permite una visión general del grupo de estudiantes que se sometieron a ella. Por otra parte, a fin de agruparlos en categorías, se generó una escala de calificación con las características indicadas en la Tabla 7.
Al procesar esta información nos encontramos con que más de la mitad del grupo, 55,4\%, presenta un nivel de lenguaje insuficiente, es decir, desconoce los símbolos, enunciados y objetos matemáticos y presenta dificultades en el cambio de registros semiótico al intentar pasar del lenguaje cotidiano al formal y viceversa. Por otra parte, el $37 \%$ del grupo presenta un nivel de competencia en el uso y conocimiento del lenguaje matemático aceptable, es decir, conoce parcialmente los elementos del lenguaje y presenta algunas dificultades en el cambio de registro semiótico. Solo un $7,6 \%$ del grupo presentó un nivel de competencia sobresaliente.

\begin{tabular}{llrr}
\hline & & F & \multicolumn{1}{c}{$\%$} \\
\hline & Insuficiente (0\%-60\%) & 51 & $55.4 \%$ \\
\multirow{3}{*}{ Nivel de lenguaje } & Aceptable (60\%- 80\%) & 34 & $37.0 \%$ \\
& Bueno (80\% - 100\%) & 7 & $7.6 \%$ \\
& Total & 92 & $100.0 \%$ \\
\hline
\end{tabular}

Tabla 7. Nivel de competencia en el conocimiento y uso del lenguaje matemático.

En general, los resultados de la Tabla 8 muestran un panorama preocupante por cuanto el nivel de complejidad de los contenidos evaluados era relativamente bajo, y consistían en un repaso de conceptos tratados en la educación básica y media. Los resultados generales de la prueba se reparten proporcionalmente cuando se considera la variable género. Tomando como parámetro la proporción muestral y obteniendo un $\mathrm{p}$-valor asociado $p>0,05$, se puede afirmar que no existen diferencias significativas en los resultados cuando se considera a los estudiantes discriminados según sean hombres o mujeres.

\subsection{Discusión}

Los resultados de esta investigación son ligeramente distintos a los de otras experiencias con universitarios. En este trabajo, en efecto, al menos un tercio de los estudiantes muestran resultados favorables en relación con el uso y conocimiento adecuado del lenguaje matemático, mientras que en otras experiencias con estudiantes de ingeniería de los primeros semestres los estudiantes competentes matemáticamente representan una proporción menor (Caputo., \& Macías, 2006; Ortega., \& Ortega, 2002; García., Segovia., \& Lupiáñez, 2011). En seguida se establecerán algunas comparaciones más concretas, pero conviene sugerir aquí la explicación del fenómeno. En efecto, el nivel mayor de competencia entre los sujetos del estudio puede estar alentado por tratarse, como se ha advertido, de una muestra de futuros docentes de matemática que, de alguna manera, están conscientes de su futuro rol. Para ellos parece estar clara la necesidad de mantener los principios de enseñanza planteados por los estándares de la National Council of Teachers of Mathematics, 2000, de acuerdo con los cuales "La enseñanza eficaz de las matemáticas requiere comprender lo que los estudiantes conocen y necesitan aprender $y$, en consecuencia, les desafía y apoya para aprender bien los nuevos conocimientos" (Godino., Batanero., \& Font, V. 2004). 
Con respecto a la identificación de símbolos, los estudiantes muestran un nivel de competencia elevado, que se demuestra en la precisión del significado que reconocen para cada símbolo y su utilización en la Teoría de Conjuntos y temas de Lógica proposicional. Esta área fue en la que los estudiantes se mostraron más fortalecidos, lo cual supone, en principio, un resultado alentador. Sin embargo, hay que considerar que se trata de docentes en formación inicial en el área de matemáticas y no del común de los estudiantes universitarios. Este fenómeno, como se ha mencionado el principio de la discusión, es inusual entre universitarios en etapa de formación inicial, pues, en efecto, lo común es que en este nivel se presenten dificultades tales como el "uso incorrecto de la notación o confusión en el uso del lenguaje simbólico y los problemas de traducción del lenguaje usual al simbólico y viceversa", así como el "desconocimiento o uso inadecuado de conceptos, definiciones o propiedades" (Caputo, \& Macías, 2006). Otras experiencias con estudiantes de ingeniería que cursan los primeros semestres en la universidad evidencian que los estudiantes no son capaces de explicar de manera satisfactoria el resultado de los símbolos matemáticos y confunden los conceptos fundamentales de matemática (Ortega., \& Ortega, 2002; García, Segovia, \& Lupiáñez, 2011). Es importante destacar que en el análisis de las relaciones bivariantes no se encontró una relación significativa entre la calidad de la respuesta y los contenidos en los que se desarrollaron los ejemplos de los símbolos empleados.

Esta situación, en la que los estudiantes muestran deficiencias en el conocimiento del lenguaje matemático y su uso, fue reiterativa en las pruebas realizadas. Algo similar reporta Morales-Urbina (2009), quien en una investigación con estudiantes universitarios que cursan en los primeros semestres la asignatura Matemática I, encontró que el conocimiento conceptual demostrado por los estudiantes en dicha prueba fue confuso (conocimientos básicos de números reales) y el conocimiento de procedimientos matemáticos, ausentes de procesos descriptivos y explicativos. Por otra parte, en cuanto al uso del conocimiento y su relación con la resolución de problemas, el presente trabajo develó que los estudiantes no poseían dominio de estrategias para resolver problemas y su nivel de conformidad con las explicaciones no superó la repetición de las mismas afirmaciones que se solicitó justificar. En este sentido se ha establecido que la identificación de símbolos obstaculiza la construcción y comprensión del lenguaje matemático y se convierte en una situación típica en estudiantes que se inician en su formación universitaria, como sostienen Caputo y Macías (2006).

En cuanto a las habilidades matemáticas para la comprensión y comunicación de sus conocimientos, los estudiantes presentaron menor dificultad al traducir lenguaje formal a lenguaje cotidiano en comparación con la relación inversa. La cualidad de la escritura formal está asociada al dominio conceptual y a la notación a través de símbolos especiales. A este respecto, los estudiantes mostraron poca habilidad para llevar expresiones formales al lenguaje cotidiano y serias dificultades para expresar con cierta precisión un enunciado del lenguaje cotidiano en lenguaje abstracto. Autores como Marquina Quintero, Moreno y Acevedo Barrios (2014) señalan que entre los estudiantes preuniversitarios esta dificultad se acentúa durante la resolución de problemas relacionados con la construcción de ecuaciones algebraicas que conlleven a una posible solución de un problema matemático. Los autores también señalan como notorio el hecho de que los errores de origen aritmético que cometen los estudiantes son transferidos al lenguaje algebraico. En esta misma dirección, implicaciones teóricas en el contexto del aprendizaje de la matemática en el inicio de la universidad tales como las presentadas por Rojano (1994), advierten de las dificultades propias del aprendizaje y uso del lenguaje matemático en comparación con el lenguaje formal. Uno de los fundamentos de su posicionamiento es que mientras que el lenguaje natural se emplea cotidianamente y se va desarrollando a diario, el lenguaje matemático solo se usa y practica en situaciones académicas y 
en escasas situaciones prácticas, lo que dificulta su perfeccionamiento.

Lo anterior muestra por qué los estudiantes colombianos, por ejemplo a la luz de los resultados de las pruebas PISA, no estén en capacidad de enfrentarse a un problema complejo de la vida real y resolverlo (Bustamante, \& Linares, 2014); además, el rendimiento de los estudiantes en pruebas estandarizadas está íntimamente ligado a la formación académica y profesional de los docentes (Giraldo, \& Quintero, 2014) por lo tanto, la formación inicial es más efectiva si los aspirantes a profesores aprenden las matemáticas universitarias de manera similar a la que se considera que sería deseable como práctica escolar y además se articula un programa por competencias donde se deben conocer los fundamentos de la disciplina matemática; su papel en la sociedad y en la cultura; su desarrollo histórico; su estructura y el modo en que las subdisciplinas que la forman están relacionadas; la importancia del lenguaje en que se comunica, entre otros (Gómez-Chacón, 2005; Riscanevo-Espitia, 2016).

\section{Conclusiones}

Con referencia a la definición de términos, se encontró que los estudiantes fallaron más en los ítems relacionados con demostraciones lógicas y evidenciaron resultados favorables cuando trabajaron con argumentos lógicos. En consecuencia, los ejercicios referidos a las demostraciones supusieron para los estudiantes un nivel de abstracción del lenguaje formal y presentaron mayor dificultad al momento de intentar demostrar equivalencias conceptuales. A pesar de este resultado diferenciado no se encontró una relación significativa entre la calidad de la respuesta y el tipo de enunciado (argumento o demostración). Ello se debe a que una parte significativa de las respuestas, relacionadas con las demostraciones, fueron consideradas como regulares.

Los resultados también evidencian una relación significativa entre la calidad de la respuesta y el tipo de traducción o cambio de registro semiótico. En consecuencia, el nivel de competencia relacionado con el conocimiento del lenguaje matemático, está asociado a la capacidad de traducción del lenguaje que presenta el estudiante.

En la identificación de objetos matemáticos los estudiantes encontraron mayores dificultades en comparación con las situaciones en las que se trataba de enunciados o símbolos matemáticos. El nivel de acierto, en forma global, se ubica en alrededor de la mitad del grupo, lo cual indica que la capacidad de los estudiantes en la comprensión de los elementos básicos del lenguaje matemático es cuestionable. Si a este hecho se suma una evaluación global, vemos que la gran mayoría del grupo presenta elementos insuficientes que se traducen en un nivel de baja competencia en el uso y conocimientos de elementos básicos del lenguaje matemático.

Los resultados sugieren reforzar el trabajo con el uso y articulación de diversos registros semióticos desde las estrategias docentes. Aunque se vislumbra parcialmente un avance en la traducción del lenguaje formal al cotidiano, los resultados dejan ver que los estudiantes se enfrentan a serias dificultades cuando deben trasladar una situación real al lenguaje formal. Por ello, un mayor énfasis en el plano curricular debería reforzar esta competencia del docente que enseña matemática.

Aunque en esta investigación la prueba estaba dirigida al diagnóstico del conocimiento y uso apropiado de la simbología y el lenguaje matemático, empleados en temas y conceptos tratados en la matemática preuniversitaria, podemos ver de forma global que, de los futuros docentes de matemática, solo una tercera parte demostró conocer y usar correctamente el lenguaje matemático. Esta deficiencia en su formación, sin duda, repercute en el desarrollo de su práctica profesional, limitando sus estrategias de enseñanza y de comunicación de los contenidos desarrollados y afectando, en consecuencia, la calidad del aprendizaje por parte de los estudiantes. 
Estos resultados sugieren analizar en una próxima investigación la forma en que los estudiantes hacen uso de la simbología y el lenguaje matemático. Descubrir la dinámica del uso de estos elementos mostraría, eventualmente, dificultades en la movilización entre registros o incluso la asociación de estas carencias a otras de carácter cultural o social.

\section{Referencias}

Aldana-Bermúdez, E., \& López-Mesa, J. (2016). Matemáticas para la diversidad: un estudio histórico, epistemológico, didáctico y cognitivo sobre perímetro y área. Revista de Investigación, Desarrollo e Innovación, 7 (1), 77-92. doi: http://dx.doi. org/10.19053/20278306.v7.n1.2016.5602

Bustamante, N., \& Linares, A. (2014). Pruebas Pisa confirmaron graves problemas del sistema educativo en Colombia. El Tiempo. Recuperado de: http://www. eltiempo.com/archivo/documento/CMS-13787115

Caputo, S., \& Macías, D. (2006). Análisis de los errores de los alumnos de la asignatura Álgebra I, al elaborar demostraciones. Fusco (Secretario General de Ciencia y Técnica), Comunicaciones Científicas y Tecnológicas. Universidad Nacional del Nordeste, Argentina. Recuperado de: http://www.unne.edu.ar/unnevieja/ Web/cyt/cyt2006/09-Educacion/2006-D-012.pdf.

Cerón-Álvarez, D. C., Mesa-Laverde, Y. C., \& Rojas-Morales, C. E. (2012). La naturaleza del conocimiento matemático y su impacto en las concepciones del profesor. Revista de Investigación, Desarrollo e Innovación, 2 (2), 49-59. Recuperado de: http://revistas.uptc. edu.co/index.php/investigacion_duitama/article/ view/1316

García, J., Segovia, I., \& Lupiáñez, J. L. (2011). Errores y dificultades de estudiantes mexicanos de primer curso universitario en la resolución de tareas algebraicas. Lupiáñez, J. L., Cañadas, M. C., Molina, M., Palarea, M., \& Maz, A. (Eds.). Investigaciones en pensamiento numérico y algebraico e historia de la matemática y educación matemática, 145-155. Granada, España: Departamento de Didáctica de la Matemá- tica. Universidad de Granada. Recuperado de http:// funes.uniandes.edu.co/2018/1/GarciaSegoviaLupianez2011.pdf

Giraldo, A. M., \& Quintero, L. V. (2014). Pruebas SABER: una perspectiva desde la educación matemática de los docentes de primaria (tesis de maestría). Universidad de Medellín. Medellín, Colombia.

Godino, J. D., Batanero, C., \& Font, V. (2004). Fundamentos de la enseñanza y el aprendizaje de las matemáticas. En Godino, J. D. (Ed.), Didáctica de las matemáticas para maestros, 5-153. Granada, España: Departamento de Didáctica de las Matemáticas. Universidad de Granada. Recuperado de: http://www. ugr.es/ jgodino/edumat-maestros/manual/9_didactica_maestros.pdf.

González-Marí, J. L. (2004). Competencias básicas en educación matemática. Málaga, España:Didáctica de la Matemática. Universidad de Málaga. Recuperado de: http://cprazuaga.juntaextremadura.net/competencias/mates/aspgenerales/Competencias_basicas_en_Educacion_Matematica\%20Gonzalez\%20 Mari.pdf.

Gómez-Chacón, I. (2005). Tendencias y retos en formación de profesores en Matemáticas. En Gómez-Chacón, I. M., \& Planchart, E. (Eds.), Educación Matemática y Formación de Profesores. Propuestas para Europa y Latinoamérica, 15-31. Bilbao: Publicaciones de la Universidad de DeustoHumanitarianNet.

Hernández, C. A. (2012). Caracterización de la actividad demostrativa en estudiantes de educación superior. Ecomatemático, 3 (1), 36 - 43.

Hernández, C. A. (2009) Caracterización de la actividad demostrativa en la Educación Superior en estudiantes de Licenciatura en Matemáticas e Informática (Tesis de maestría). Universidad Nacional Experimental del Táchira, Venezuela.

Marquina-Quintero, J. R., Moreno, G. A., \& Acevedo-Barrios, A. A. (2014). Transformación del lenguaje 
natural al lenguaje algebraico en educación media general. Educere, 18 (59), 119 - 132.

Méndez, A. I. (2007). Metodologías y técnicas de investigación aplicadas a la comunicación. Maracaibo, Venezuela: Ediciones del Vice Rectorado Académico. Universidad del Zulia.

Ministerio de Educación Nacional. (2006a). ¿Qué hay que saber de las Competencias Matemáticas? Portal Colombia Aprende: Ministerio de Educación Nacional. Recuperado de: http://www.colombiaaprende. edu.co/html/home/1592/article-103987.html

Ministerio de Educación Nacional. (2006b). Estándares básicos de competencias en lenguaje, matemáticas, ciencias y ciudadanía. Bogotá, Colombia: Ministerio de Educación Nacional.

Morales-Urbina, E. M. (2009). Los conocimientos previos y su importancia para la comprensión del lenguaje matemático en la educación superior. Universidad, ciencia y tecnología. 13 (52), 211-222

National Council of Teachers of Mathematics. (2003). Principios y estándares para la educación matemática. Sociedad Andaluza de Educación Matemática (Fernández, M., trad.). Sevilla, España: SAEM Thales. Recuperado de: http://revistasuma.es/IMG/pdf/48/105112.pdf.

National Council of Teachers of Mathematics. (2000). Principles and Standards for School Mathematics. Reston. VA: National Council of Teachers of Mathematics.

Niss, M. (1999). Aspects of the nature and state of research in mathematics education. Educational studies in mathematics, 40 (1), 1-24.

Niss, M. (2003). Mathematical competencies and the learning of mathematics: The Danish KOM project. En 3rd Mediterranean conference on mathematical education. Recuperado de: http://equipsice.uab.cat/ file.php/28/Compet_Niss.pdf 115-124.

Ortega, J. F., \& Ortega, J. A. (2002). Experiencia sobre el conocimiento del lenguaje matemático. En
Asociación Española de Profesores Universitarios de Matemáticas para la Economía y la Empresa. Comunicación llevada a cabo en las $X$ Jornadas ASEPUMA 2002. Universidad de Valencia, España. Recuperado de: http://www.uv.es/asepuma/X/I17C.pdf

Piratoba-Gil, R. P., \& Rojas-Morales, C. E. (2014). Cambios en las concepciones iniciales e inducidas sobre la naturaleza de las matemáticas y su didáctica, en estudiantes de un programa de Licenciatura en Matemáticas y Estadística. Revista de Investigación, Desarrollo e Innovación, 5 (1), 32-45. doi: https://doi. org/10.19053/20278306.3140

Riscanevo-Espitia, L. (2016). La teoría de la práctica social del aprendizaje en la formación de profesores de matemáticas. Revista de Investigación, Desarrollo e Innovación, 7 (1), 93-110. doi: https://doi. org/10.19053/20278306.v7.n1.2016.5635

Rojano, T. (1994). La matemática escolar como lenguaje. Nuevas perspectivas de investigación y enseñanza. Enseñanza de las ciencias, 12 (1), 45-56.

Sepúlveda-Delgado, O. (2015). Estudio del conocimiento didáctico - matemático del profesor universitario: un marco teórico de investigación. Revista de Investigación, Desarrollo e Innovación, 6 (1), 29-43. doi: http://doi.org/10.19053/20278306.4048

Vargas, C., \& Giménez, J. (2012). Competencia comunicativa y formación docente. En Font, V., Giménez, J., Larios, V., \& Zorrilla, J. F. (coord.). Competencias del profesor de matemática de secundaria y bachillerato. Barcelona, España: Ediciones Universitat Barcelona, 103-114.

Vilaró, R. (2005). Desafíos de la formación docente ante la realidad social y la sociedad del conocimiento. En Gómez-Chacón, I. M., \& Planchart, E. (Eds.), Educación Matemática y Formación de Profesores. Propuestas para Europa y Latinoamérica, 51-71. Bilbao: Publicaciones de la Universidad de DeustoHumanitarianNet. 